\title{
Controlling atomic structures and photoabsorption processes by an infrared laser
}

\author{
X. M. Tong ${ }^{1,2, *}$ and N. Toshima ${ }^{1}$ \\ ${ }^{1}$ Institute of Materials Science, Graduate School of Pure and Applied Sciences, University of Tsukuba, 1-1-1 Tennodai, \\ Tsukuba, Ibaraki 305-8573, Japan \\ ${ }^{2}$ Center for Computational Sciences, University of Tsukuba, 1-1-1 Tennodai, Tsukuba, Ibaraki 305-8577, Japan
}

(Received 9 April 2010; published 7 June 2010)

\begin{abstract}
We propose a theoretical method to calculate the infrared (IR) laser-assisted photoabsorption cross sections over a broad energy range by a single calculation. In this method we define an initial wave function as the product of the dipole operator and the atomic ground state, propagate the initial wave function in the IR laser field with different initial phases, then calculate the generalized autocorrelation function, which is defined as the averaged value of the autocorrelation function over the initial phase from 0 to $2 \pi$ (or one IR optical cycle). The IR laser-assisted photoabsorption cross sections are obtained by the Fourier transform of the generalized autocorrelation function. We apply this method to study the IR laser-assisted photoabsorption of He atoms. From the simulation results, we see that the IR laser field affects not only the resonant position but also the lifetime or the width of the bound states. This photoabsorption cross section is an important quantity to analyze the IR-laser-assisted dynamical processes by an attosecond pulse, a pulse train, or a free-electron laser.
\end{abstract}

DOI: 10.1103/PhysRevA.81.063403

PACS number(s): 32.80.Qk, 32.80.Rm

\section{INTRODUCTION}

To understand and control natural phenomena is one of the main goals of the physical sciences. Photoabsorption spectra have been used for a long time to study atomic structures and identify the makeup of a material [1]. One can control atomic processes by applying static electric fields [2,3], static magnetic fields [4,5], or crossed static electric and magnetic fields [6,7]. As the laser technology advances, one can control even the photoabsorption of atoms in a very short time scale with the assistance of a moderately intense infrared (IR) laser field by a noncoherent light source from the synchrotron radiations [8-10] or by a coherent light source from the attosecond pulse (AP) or attosecond pulse train (APT) [11,12]. The IR field modifies the atomic structure and the photoabsorption process, for example, the IR-field-induced transparency for $\mathrm{x}$ rays $[13,14]$. For most of the moderately intense IR-field-assisted processes, the IR field only modifies the energy structures of the excited states or continuum without perturbing the ground-state wave function. Recently, by using the IR-assisted photoexcitation process by the APT, Swodoba et al. [15] could even investigate the IR-field-induced energy shift of a specified excited state. A recent study [16] suggested a mechanism to understand the IR-assisted photoabsorption by the AP or APT. According to this theory, Floquet states are formed when atoms are subjected to a moderately intense IR field. For a given Floquet state, there are many sidebands separated evenly with one IR photon energy. The energy structure of a Floquet state looks like a multislit in the energy domain. With this understanding, we proposed a way to control the slit structures by tuning the IR laser intensity and to control the photoabsorption process by tuning the relative field strengths of the extreme ultraviolet light source at different frequencies, which is equivalent to tuning the current strengths going through different slits. In such a way the IR-assisted ionization yield of $\mathrm{He}$ of the APT can be enhanced or suppressed significantly by tuning the relative phase between the IR laser and the APT [17]. To effectively control the IR-assisted photoabsorption process, we need the IR-assisted photoabsorption cross section. Such cross sections can be calculated by solving the time-dependent Schrödinger equation at each given photon energy [17] or solving a time-independent Floquet-type equation [18]. The disadvantage of these methods is that they take a lot of computational time. For example, the computational time increases cubically as the number of the Floquet blocks increases if we solve the time-independent Floquet equations. To reduce the numerical burden, we propose a method to study the photoabsorption processes more effectively. In this method, we calculate the IR-assisted photoabsorption cross sections by the Fourier transformation of a generalized autocorrelation function. The method is equivalent to the time-independent Floquet method [19] but the details of the Floquet wave function are not explicitly required. The computational time increases only linearly in the present method as the number of the Floquet blocks increases in proportion to the number of initial phases of the IR field. The proposed method is more efficient from the viewpoint of the computational time and the storage size.

\section{THEORETICAL METHOD}

When an atom is placed in a periodic external field, its motion is governed by the following time-dependent Schrödinger equation (atomic units $\hbar=m=e=1$ are used unless stated otherwise):

$$
i \frac{\partial \Psi(t)}{\partial t}=H(t) \Psi(t)
$$

with $H(t)=H_{0}+V(t)$, where $H_{0}$ is the atomic external fieldfree Hamiltonian and $V(t)$ is periodic in $T$ as $V(t+T)=$ $V(t)$. Based on the Floquet theory [19], the solution of the above equation can be generally expressed as

$$
\Psi_{\alpha}(t)=e^{-i \epsilon_{\alpha} t} \sum_{n=-\infty}^{\infty} e^{-i n \omega t} \phi_{\alpha, n},
$$


where $\epsilon_{\alpha}$ is the quasienergy, $\phi_{\alpha, n}$ is the time-independent wave function of the $n$th Fourier component of the $\alpha$ Floquet state, and $\omega=\frac{2 \pi}{T}$ is the frequency of the periodic external field. If the periodic field is strong enough to modify the structures of the exited and continuum states but weak enough not to perturb the ground state, the photoabsorption cross section from the ground state to the Floquet states can be expressed as

$$
\sigma\left(\omega_{p}\right)=\frac{4 \pi \omega_{p}}{c} \operatorname{Im} \sum_{\alpha, n} \frac{\left|\left\langle\Psi_{g}|\mathbf{d}| \phi_{\alpha n}\right\rangle\right|^{2}}{\left(\epsilon_{\alpha}+n \omega\right)-\left(\epsilon_{g}+\omega_{p}\right)-i \eta},
$$

where $\eta$ is an infinitesimal positive number to represent adiabatical switching on of the probe light, $\omega_{p}$ is the frequency of the probe light, $\mathbf{d}$ is the dipole operator, $\Psi_{g}$ is the ground-state wave function, and $\epsilon_{g}$ is the ground-state energy. If there is no periodic external field, there is only one nonzero Fourier component for a given Floquet state and the above equation turns to the standard one for photoabsorption without the periodic field [1]. In the traditional procedure, we obtain the time-dependent wave function $\Psi_{\alpha}(t)$ by solving the time-independent Floquet equation. The computational load increases cubically as the number of the Floquet blocks increases and the applicability of the method to real systems is limited. If many continuum states are involved, as is the case for the IR laser-assisted photoabsorption process, the problem becomes even more difficult and more complicated.

We developed a theoretical method to study the photoabsorption process in an oscillating magnetic field for a nondegenerate system [20]. The previous method works only for a system of nondegenerate states and this limits the application of the method. In this paper we extend the method to a general case in which both bound and continuum degenerate states are involved. For such a purpose, we define the time-dependent Hamiltonian as $H(t, \tau)=H_{0}+V(t+\tau)$, with $\tau$ a time shift that corresponds to an initial phase $\delta=\frac{2 \pi \tau}{T}$, and set the initial wave function $|\Psi(t=0, \tau)\rangle=\mathbf{d}\left|\Psi_{g}\right\rangle$. We solve the time-dependent wave function $\Psi(t, \tau)$ for a given $H(t, \tau)$ and define a generalized autocorrelation function as

$$
C(t)=\frac{1}{T} \int_{0}^{T}\langle\Psi(t=0, \tau) \mid \Psi(t, \tau)\rangle d \tau .
$$

The photoabsorption cross section can then be represented as

$$
\sigma\left(\omega_{p}\right)=\frac{4 \pi \omega_{p}}{c} \operatorname{Im} \int_{0}^{\infty} i C(t) e^{i\left(\omega_{p}+\epsilon_{g}\right) t-\eta t} d t .
$$

In the practical simulation, we confine the numerical calculation in a finite space and have to filter out the wave function in the outer region using an energy-dependent optical potential $[21,22]$. Generally speaking, the quasienergy $\epsilon_{\alpha}$ in Eq. (2) is a complex number with the imaginary part representing the width of the Floquet state. To avoid the divergency at $t \rightarrow-\infty$ in Eq. (5), we adiabatically switch off the probe field instead of adiabatically switching it on.

Now let us prove the equivalence of Eqs. (3) and (5). Since the Floquet states of Eq. (2) form a complete set in the composite Hilbert space, the initial state $\mathbf{d}\left|\Psi_{g}\right\rangle$ can be expanded in terms of the Floquet states as

$$
\mathbf{d}\left|\Psi_{g}\right\rangle=\sum_{\alpha} C_{\alpha}^{\tau}\left|\Psi_{\alpha}(t=0, \tau)\right\rangle
$$

with the coefficient

$$
C_{\alpha}^{\tau}=\sum_{n} e^{i n \omega \tau}\left\langle\phi_{\alpha, n}|\mathbf{d}| \Psi_{g}\right\rangle
$$

and the time-dependent wave function can be expressed as

$$
\begin{aligned}
\Psi(t, \tau) & =\sum_{\alpha} C_{\alpha}^{\tau} \Psi_{\alpha}(t, \tau) \\
& =\sum_{\alpha, m, n} e^{-i\left(\epsilon_{\alpha}+m \omega\right) t} e^{i(n-m) \omega \tau}\left\langle\phi_{\alpha, n}|\mathbf{d}| \Psi_{g}\right\rangle \phi_{\alpha, m} .
\end{aligned}
$$

By using

$$
\frac{1}{T} \int_{0}^{T} e^{i(n-m) \omega \tau} d \tau=\delta_{n, m}
$$

we get

$$
\begin{aligned}
C(t) & =\frac{1}{T} \int_{0}^{T}\left\langle\Psi_{g}|\mathbf{d}| \Psi(t, \tau)\right\rangle d \tau \\
& =\sum_{\alpha, n} e^{-i\left(\epsilon_{\alpha}+n \omega\right) t}\left|\left\langle\phi_{\alpha, n}|\mathbf{d}| \Psi_{g}\right\rangle\right|^{2} .
\end{aligned}
$$

The Fourier transform of Eq. (10) gives Eq. (3) if the propagation time $t_{\max }$ approaches infinity. In the simulation, we choose the infinitesimal number as $\eta=\frac{5}{t_{\max }}$. By varying the propagation time $t_{\max }$, we can confirm the numerical convergency and obtain the natural width and the lifetime of a resonant state in the laser field. Such information can be obtained in the two-photon ionization experiment as shown in a recent work [15].

\section{RESULTS AND DISCUSSION}

The method discussed in Sec. II is very general and it can be applied to any system in a periodic external field. Unlike our previous works [16,17], this method even works for the systems for which the asymptotic wave function is unknown analytically. In this paper, we study the IR-assisted photoabsorption process of $\mathrm{He}$ at different intensities for an 800-nm IR laser. The atomic Hamiltonian can be written as $H_{0}=-\frac{1}{2} \nabla^{2}+V_{\text {eff }}(r)$ with a model potential $V_{\text {eff }}(r)$ [23] describing the electron parent-core interaction. The IR field interaction is written as $V(t, \tau)=-z E_{0} \cos (\omega t+\delta)$, where $z$ is the electron $z$ coordinate, $E_{0}$ is the IR field strength, and $\delta=\frac{2 \pi \tau}{T}$ is the initial phase. We assume that both the IR laser and the probe light are linearly polarized along the $z$ direction. The time-dependent wave function $\Psi(t, \tau)$ is obtained from Eq. (1) by using the split-operator method with the generalized pseudospectral grid in the energy representation [24] as

$|\Psi(t+\Delta t, \tau)\rangle=e^{-i H_{0} \Delta t / 2} e^{-i V(t, \tau) \Delta t} e^{-i H_{0} \Delta t / 2}|\Psi(t, \tau)\rangle$

with initial wave function $|\Psi(t=0, \tau)\rangle=\mathbf{d}\left|\Psi_{g}\right\rangle$. Instead of projecting the wave function in the outer region as our previous work [16], we apply an energy-dependent optical potential [25] to absorb the wave function in the outer region. With the calculated $|\Psi(t+\Delta t, \tau)\rangle$, we obtain the generalized autocorrelation function from Eq. (4) and then the photoabsorption cross section from Eq. (5). In the present simulation, we use 512 points in the radial part and 40 partial waves with 128 equal distribution $\tau$ from $0 \rightarrow T$. This 


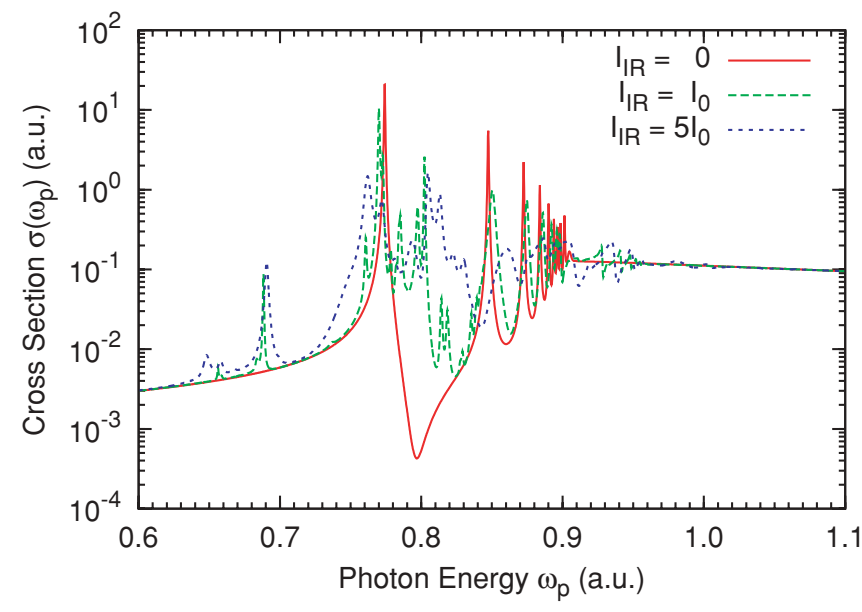

FIG. 1. (Color online) The IR-assisted photoabsorption cross sections of He at three IR intensities, 0 (solid line), $I_{0}$ (dashed line), and $5 I_{0}$ (dotted line), respectively, with $I_{0}=10^{12} \mathrm{~W} / \mathrm{cm}^{2}$.

corresponds to 128 Floquet blocks with more than a $2 \times 10^{4}$ atomic basis set. Diagonalizing a $10^{6} \times 10^{6}$ matrix is still a great task for a supercomputer. We have varied the propagation time from 128 to 512 o.c. (the IR optical cycles) to check the convergency. In the following we present the results of propagation time 256 o.c. unless stated otherwise.

Figure 1 shows the IR-assisted photoabsorption cross sections at three different IR intensities. Without the IR field, the photoabsorption spectra are divided into two parts, photoexcitation below the ionization threshold and photoionization above the threshold. The photoexcitation consists of many sharp resonances and the energy widths of the resonances are very narrow. The widths in the figure do not have significant physical meaning since the propagation time is finite. The longer propagation time is, the sharper the resonant peaks are. For photoionization, the spectra change smoothly as a function of the photon energy and they are insensitive to the propagation time. As we switch on the IR laser, the atomic structures are described by the Floquet states and there are many sidebands for a bound state. For the photoionization far above the ionization threshold, the spectra depend on the IR intensity weakly.

There are some structures near the ionization threshold and they are attributed to the sidebands of the bound states. The strengths of the sideband contribution increase as the IR intensity increases. The ionization potential also increases as the IR intensity increases owing to the ponderomotive shift $[26,27]$.

The effect of the ponderomotive shift can be seen more clearly in Fig. 2. The ionization threshold increases linearly as the IR intensity increases and it is well represented by $I_{p}+U_{p}$, with $I_{p}$ the atomic ionization potential and $U_{p}$ the ponderomotive energy of a free electron in the laser field. The ponderomotive shift mainly affects the highly excited states and the energy shifts are well represented by the ponderomative energy. This is the reason why the abovethreshold-ionization (ATI) peaks from the highly excited states (the so-called Freeman resonances [28]) do not depend on the IR intensity. For the lower bound states, such as the $2 p$ state, the shift is negligibly small. From the energy difference, we can

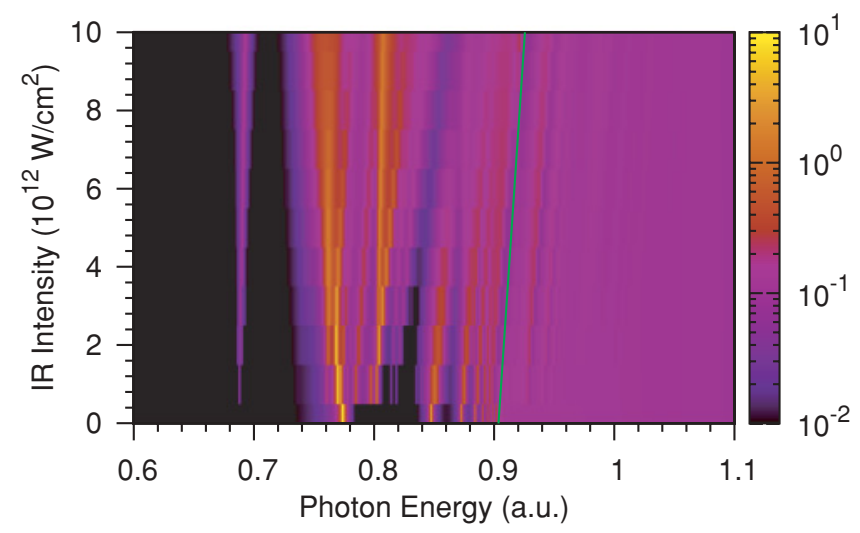

FIG. 2. (Color online) The IR-assisted photoabsorption cross sections of $\mathrm{He}$ as a function of the IR intensity. The solid line shows the ionization threshold due to the ponderomotive shift.

classify the origin of the sharp ATI peak. As the IR intensity increases, we do not see a peak structure clearly for the highly excited states because the width of the highly excited states in the IR field is comparable to or even larger than the energy separation between the different excited states.

Without the IR field, the lifetimes of the excited states are very long in the femtosecond or even in the picosecond time scale. So the photoabsorption widths of the excited states are very narrow. The IR field affects not only the absorption cross sections but also the lifetime of the excited states. In the present calculations the width of a resonance comes from two parts, the natural width due to the IR field and the artificial width due to the propagation time. By varying the propagation time $t_{\max }$ we can extract the natural width if it is comparable to or larger than the artificial one.

Figure 3 shows the IR-assisted photoabsorption cross sections at $5 I_{0}\left(I_{0}=10^{12} \mathrm{~W} / \mathrm{cm}^{2}\right)$ with different propagation times. The patterns of the photoabsorption spectra are not sensitive to the propagation time, especially for the highly excited states. For the lower resonant states, the peak value

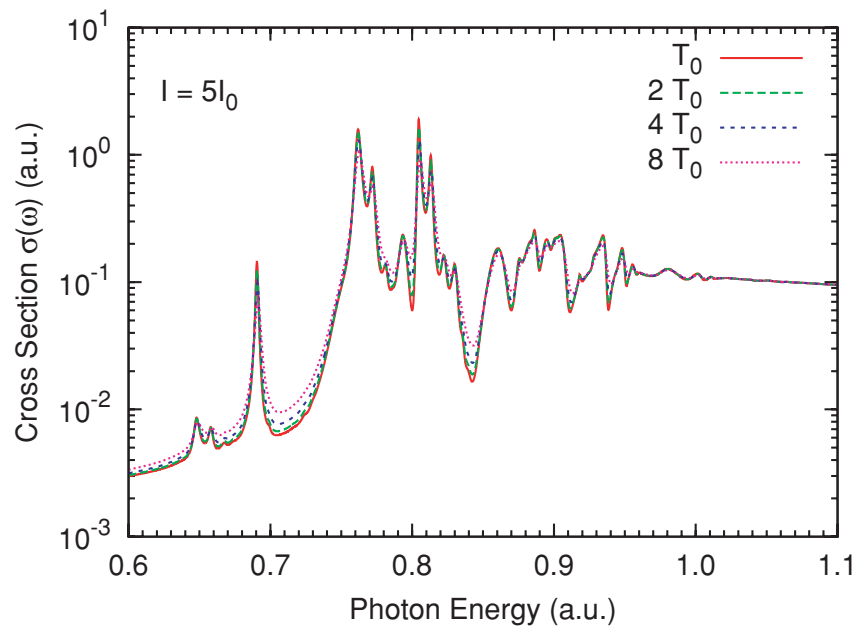

FIG. 3. (Color online) The IR-assisted photoabsorption cross sections of He calculated with different propagation times in units of $T_{0}, 2 T_{0}, 4 T_{0}$, and $8 T_{0}$ with $T_{0}=64$ o.c. (o.c. denotes the IR optical cycle). 


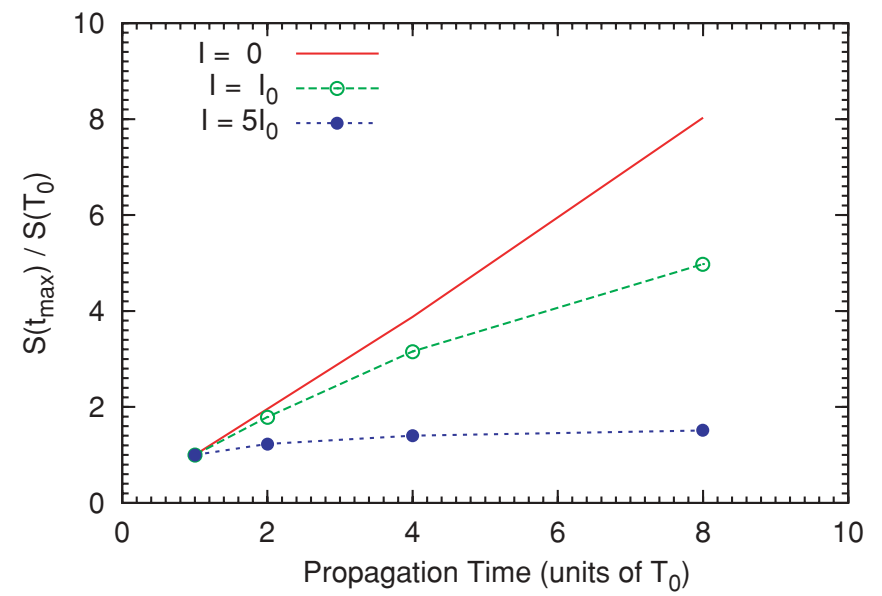

FIG. 4. (Color online) The ratios $S\left(t_{\max }\right) / S\left(T_{0}\right)$ of the IR-assisted photoabsorption cross sections of He for the $1 s \rightarrow 2 p$ transition calculated with different propagation times at three IR intensities.

comes to depend on the propagation time. Denoting the natural width by $\Gamma$, the peak value is proportional to $S\left(t_{\max }\right) \propto$ $\left(\frac{\Gamma}{2}+\frac{5}{t_{\max }}\right)^{-1}$. If we choose $T_{0}=64$ o.c. and plot $S\left(t_{\max }\right) / S\left(T_{0}\right)$ for the $1 s \rightarrow 2 p$ transition as shown in Fig. 4, we can extract the actual width of the $2 p$ state at different IR intensities. Without the IR laser, the natural width of $2 p$ is very narrow in comparison to the propagation time so that the peak value is almost linearly proportional to the propagation time as shown in Fig. 4. At the highest intensity $5 I_{0}$, the $S$ value is almost flat. This means that the artificial width introduced from the propagation time is much smaller. The natural width and the artificial width are comparable to each other at $I_{0}$ and we can fit the above relation to get the natural width. For the highly excited states, the natural width increases quickly as the IR intensity increases and we can get the natural width for a reasonable propagation time.

To summarize, we proposed a theoretical method which can be used to study the IR-assisted photoabsorption processes. The advantages of the method are (1) the cross sections over a broad photon-energy range can be obtained in a single calculation and (2) in the calculations we do not need the detailed information of the Floquet wave function. The calculated cross sections also provide us with a guide to investigate how to control the photoabsorption process by tuning the energy range of AP or APT. Using the free-electron laser, one can also directly measure the IR-assisted photoabsorption cross sections and control the dynamical processes by tuning the IR intensity.

\section{ACKNOWLEDGMENTS}

This research was supported by Grand-in-Aid for Scientific Research (C) from the Japan Society for the Promotion of Science. The authors would like to thank Professor C. L. Cocke and Dr. P. Ranitovic for their useful discussions.
[1] U. Fano and J. W. Cooper, Rev. Mod. Phys. 40, 441 (1968).

[2] J. R. Harries, J. P. Sullivan, J. B. Sternberg, S. Obara, T. Suzuki, P. Hammond, J. Bozek, N. Berrah, M. Halka, and Y. Azuma, Phys. Rev. Lett. 90, 133002 (2003).

[3] X. M. Tong and C. D. Lin, Phys. Rev. Lett. 92, 223003 (2004).

[4] A. Holle, G. Wiebusch, J. Main, G. Z. K. H. Welge, G. Wunner, T. Ertl, and H. Ruder, Z. Phys. D 5, 279 (1987).

[5] S. I. Chu and X. M. Tong, Chem. Phys. Lett. 294, 31 (1998).

[6] G. Wiebusch, J. Main, K. Kruger, H. Rottke, A. Holle, and K. H. Welge, Phys. Rev. Lett. 62, 2821 (1989).

[7] X. M. Tong and Shih-I Chu, Phys. Rev. A 61, 031401(R) (2000).

[8] M. P. Hertlein, H. Adaniya, J. Amini, C. Bressler, B. Feinberg, M. Kaiser, N. Neumann, M. H. Prior, and A. Belkacem, Phys. Rev. A 73, 062715 (2006).

[9] L. Young et al., Phys. Rev. Lett. 97, 083601 (2006).

[10] S. H. Southworth et al., Phys. Rev. A 76, 043421 (2007).

[11] P. Johnsson, J. Mauritsson, T. Remetter, A. L'Huillier, and K. J. Schafer, Phys. Rev. Lett. 99, 233001 (2007).

[12] P. Ranitovic et al., New J. Phys. 12, 013008 (2010).

[13] S. E. Harris, J. E. Field, and A. Imamoğlu, Phys. Rev. Lett. 64, 1107 (1990)

[14] C. Buth, R. Santra, and L. Young, Phys. Rev. Lett. 98, 253001 (2007).
[15] M. Swoboda, T. Fordell, K. Klünder, J. M. Dahlström, M. Miranda, C. Buth, K. J. Schafer, J. Mauritsson, A. L'Huillier, and M. Gisselbrecht, Phys. Rev. Lett. 104, 103003 (2010).

[16] X. M. Tong, P. Ranitovic, C. L. Cocke, and N. Toshima, Phys. Rev. A 81, 021404(R) (2010).

[17] X.-M. Tong and N. Toshima, Phys. Rev. A 81, 043429 (2010).

[18] C. Buth and R. Santra, Phys. Rev. A 75, 033412 (2007).

[19] S. I. Chu and D. A. Telnov, Phys. Rep. 390, 1 (2004).

[20] T. Shirahama, X.-M. Tong, K. I. Hino, and N. Toshima, Phys. Rev. A 80, 043414 (2009).

[21] J. Perie and G. Jolicard, J. Phys. B 26, 4491 (1993).

[22] X. M. Tong, K. Hino, and N. Toshima, Phys. Rev. Lett. 97, 243202 (2006).

[23] X. M. Tong and C. D. Lin, J. Phys. B 38, 2593 (2005).

[24] X. M. Tong and S. I. Chu, Chem. Phys. 217, 119 (1997).

[25] X. M. Tong, T. Shirahama, K. Hino, and N. Toshima, Phys. Rev. A 75, 052711 (2007).

[26] M. D. Davidson, J. Wals, H. G. Muller, and H. B. van Linden van den Heuvell, Phys. Rev. Lett. 71, 2192 (1993).

[27] E. S. Toma, H. G. Muller, P. M. Paul, P. Breger, M. Cheret, P. Agostini, C. Le Blanc, G. Mullot, and G. Cheriaux, Phys. Rev. A 62, 061801(R) (2000).

[28] R. R. Freeman, P. H. Bucksbaum, H. Milchberg, S. Darack, D. Schumacher, and M. E. Geusic, Phys. Rev. Lett. 59, 1092 (1987). 